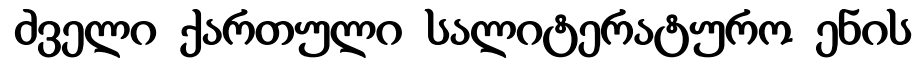

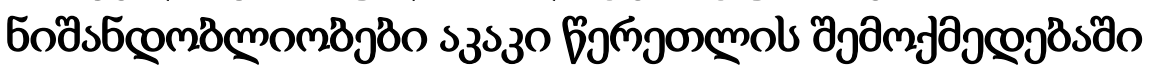

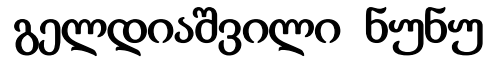

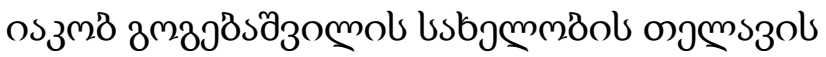

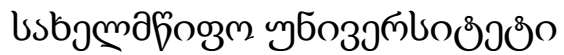

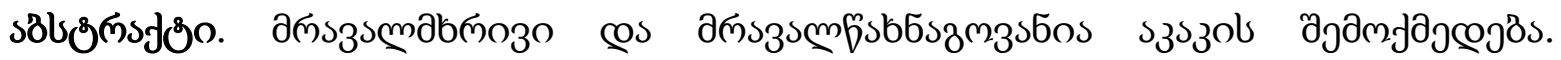

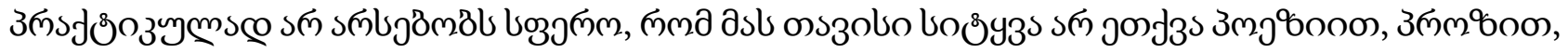

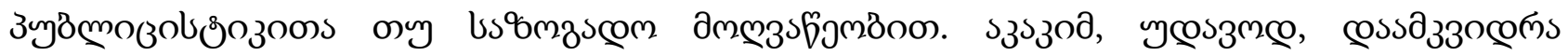

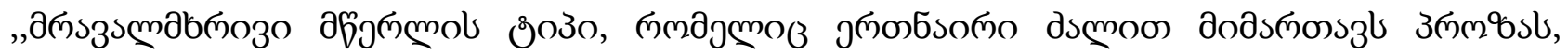

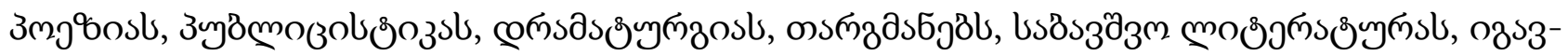

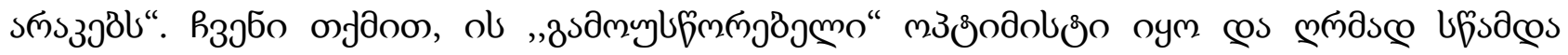

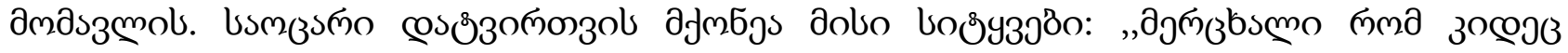

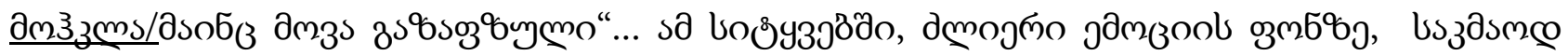

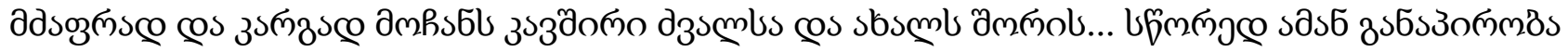

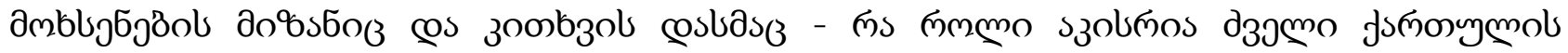

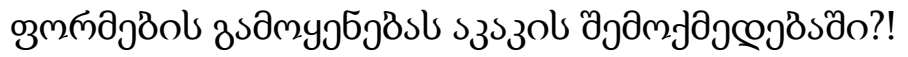

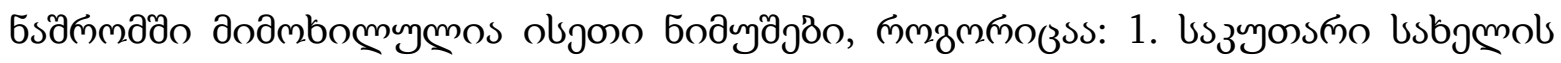

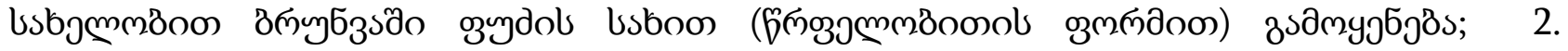

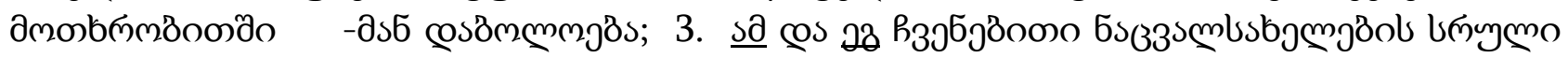

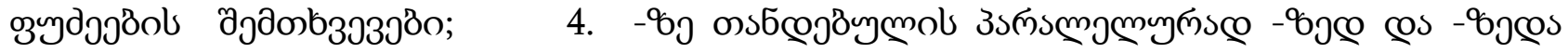

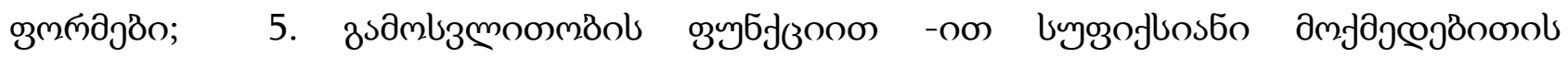

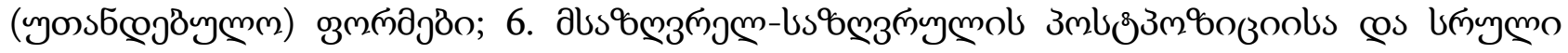

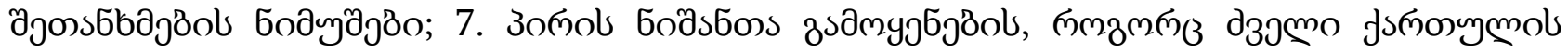

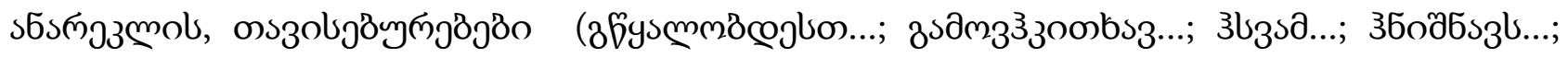

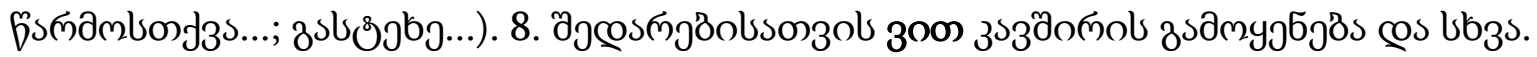

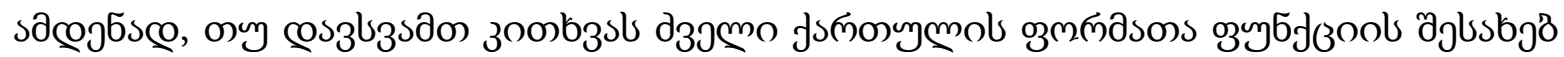

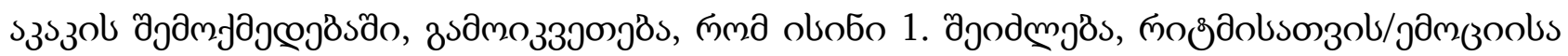

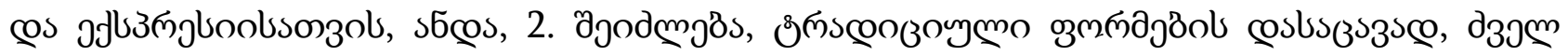

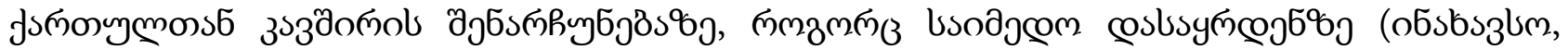

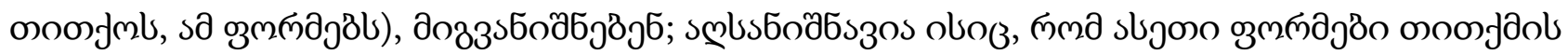

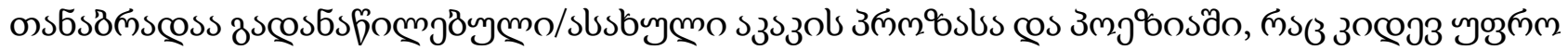

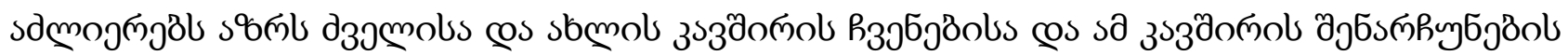

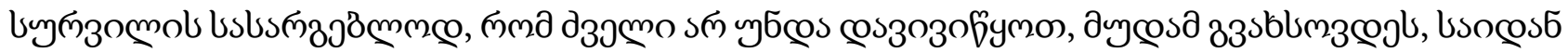

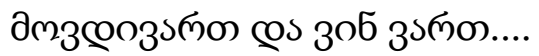

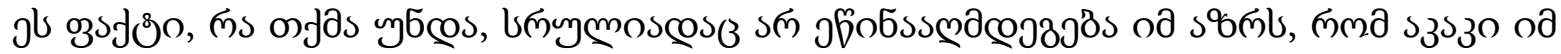

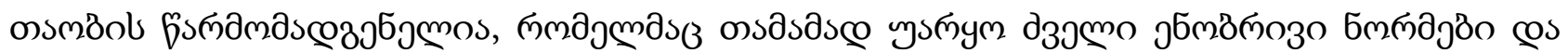




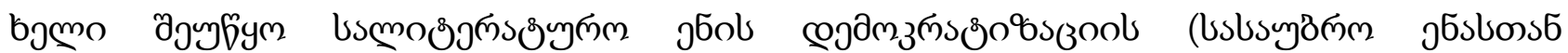

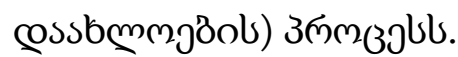

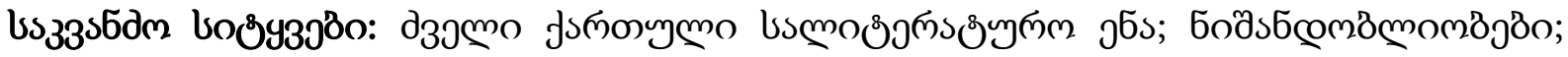

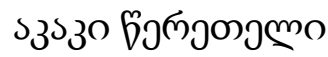

\section{gjlsзsmo.}

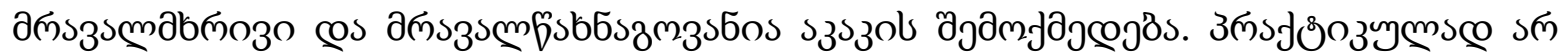

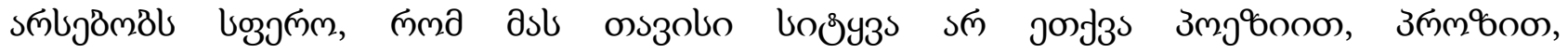

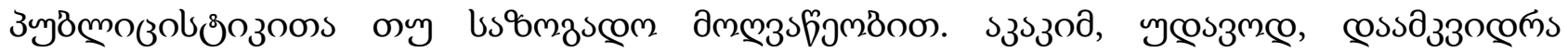

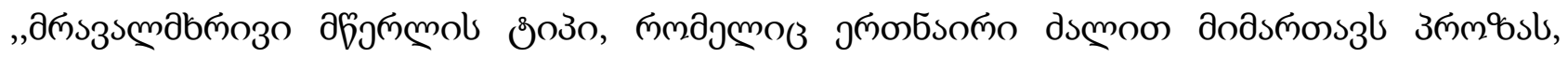

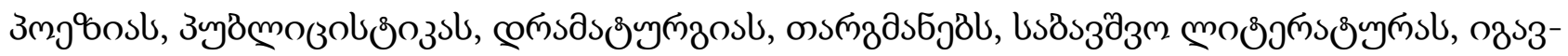

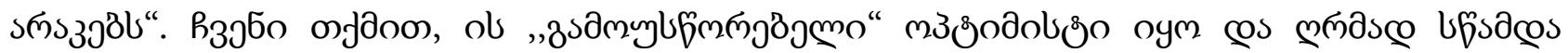

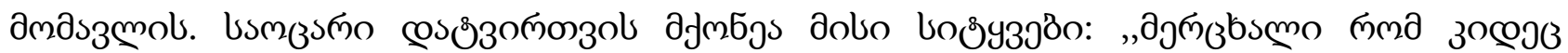

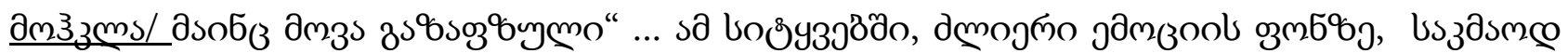

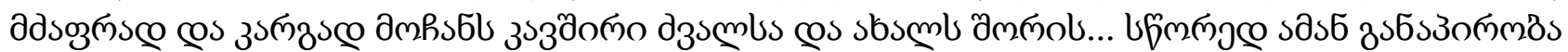

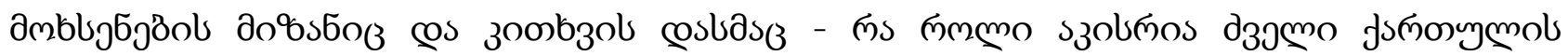

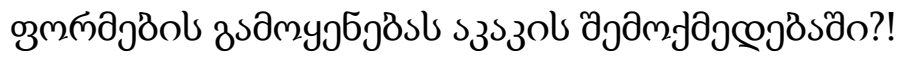

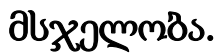

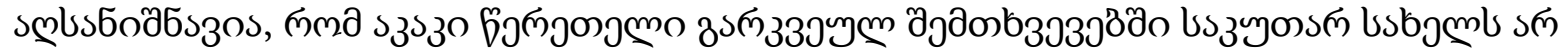

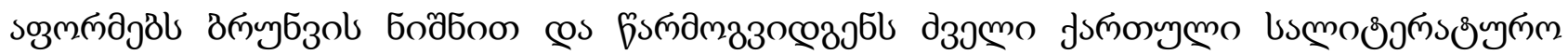

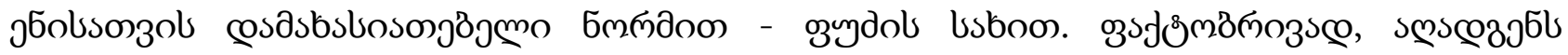

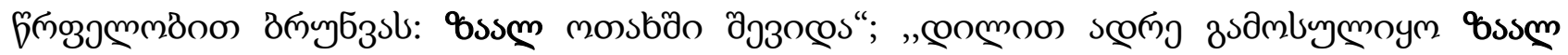

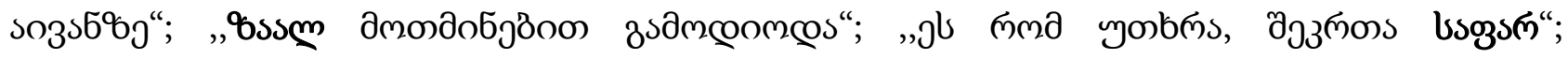

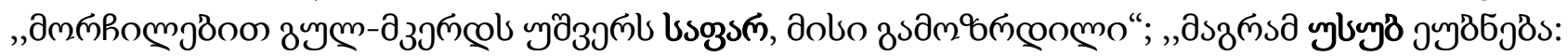

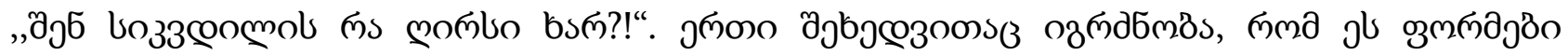

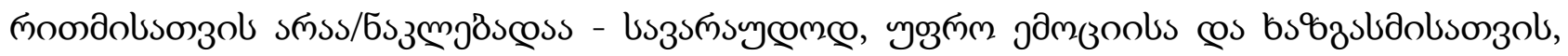

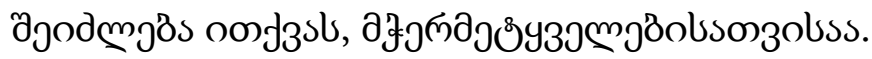

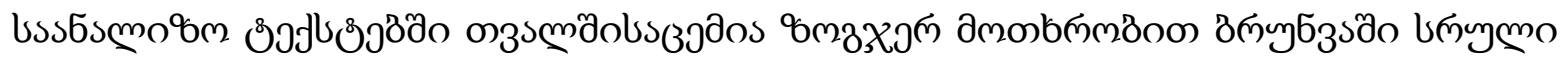

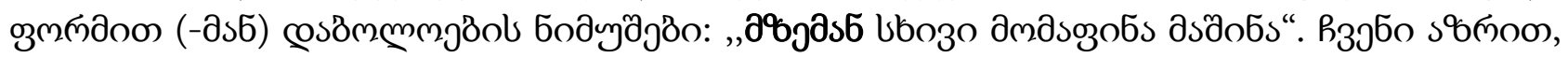

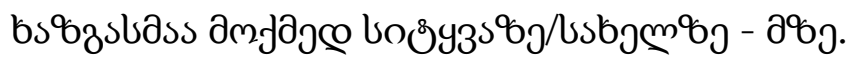

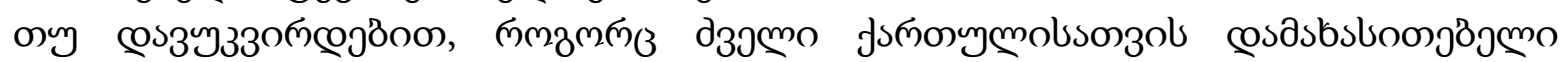

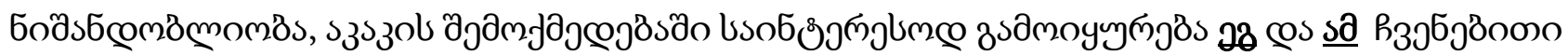

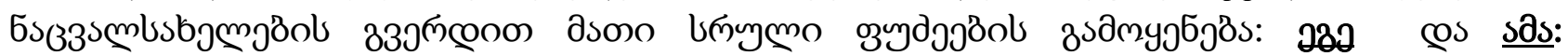

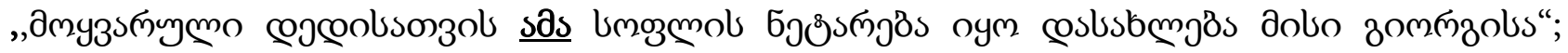

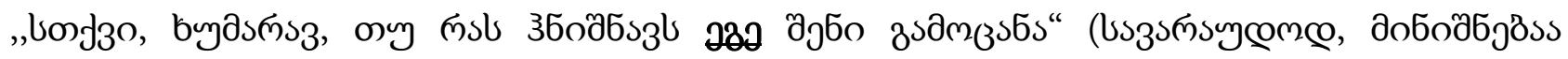

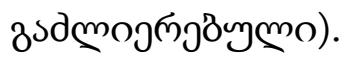

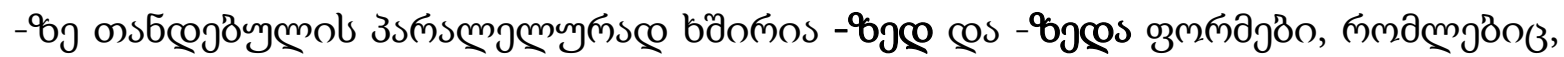

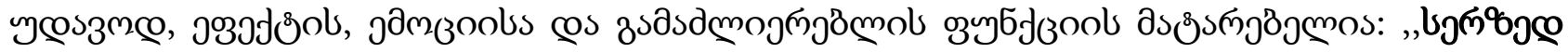

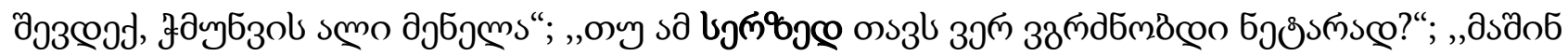

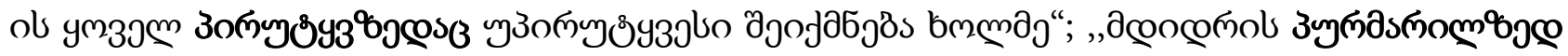

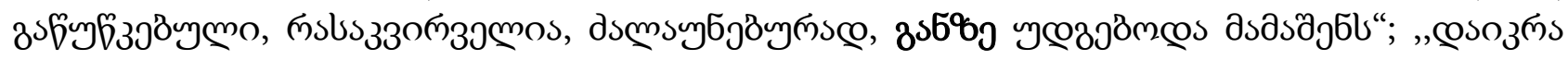

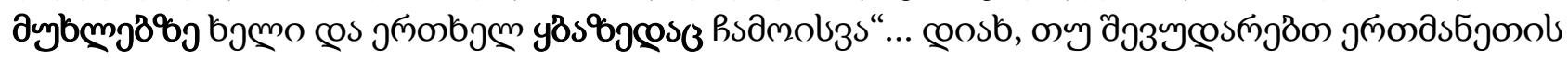

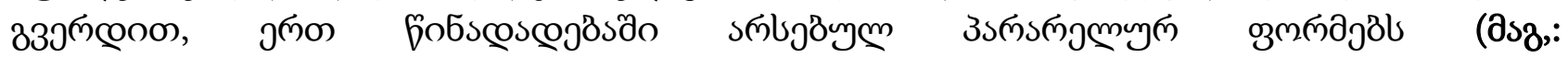




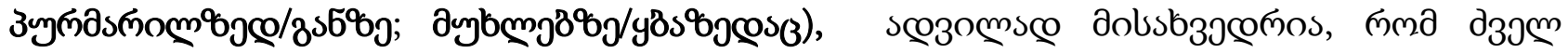

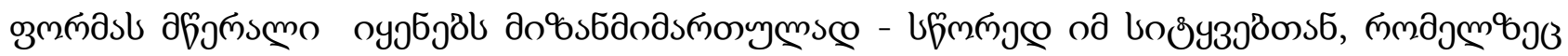

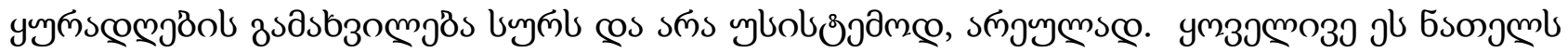

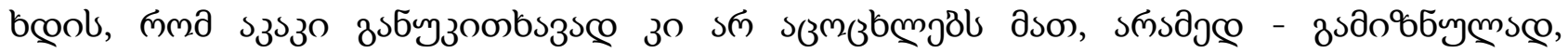

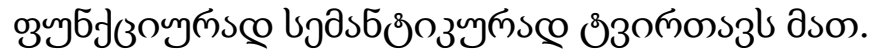

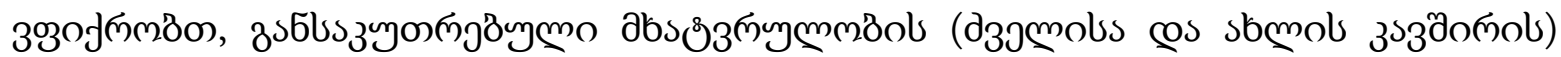

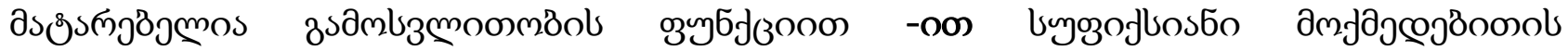

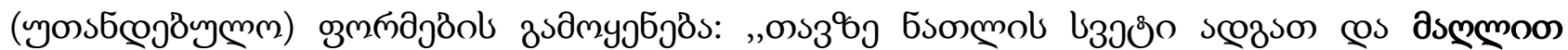

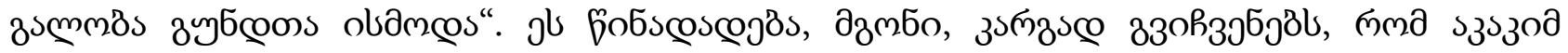

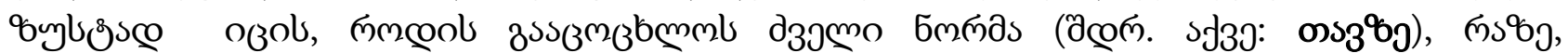

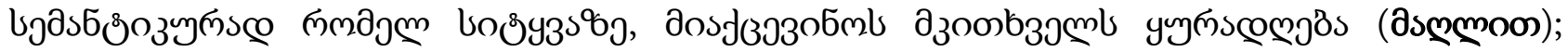

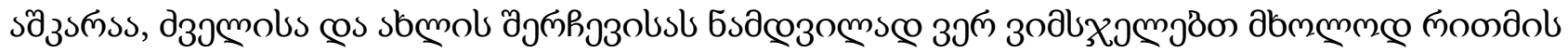

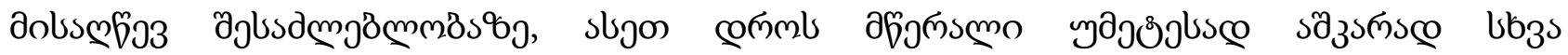

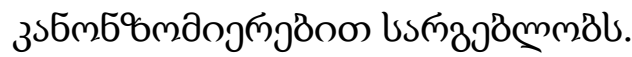

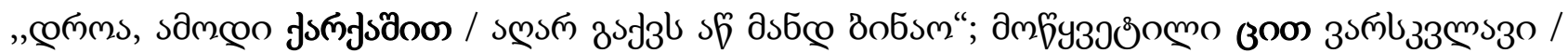

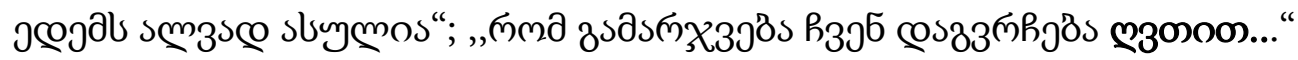

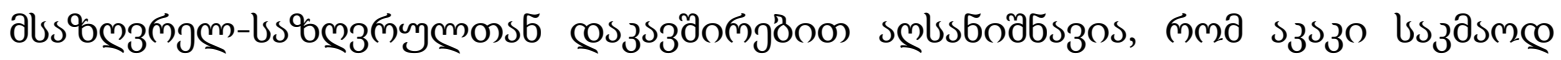

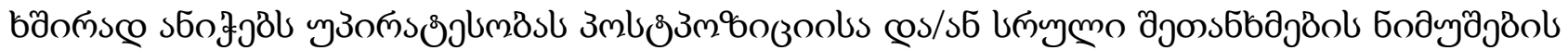

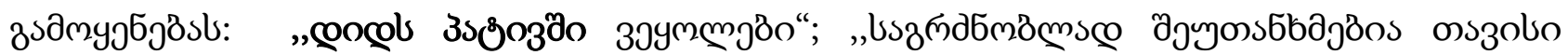

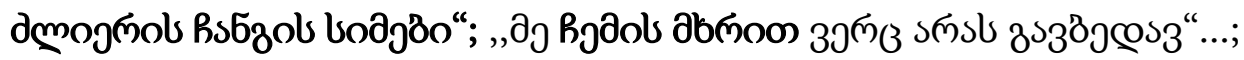

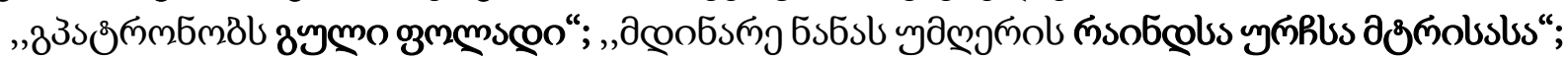

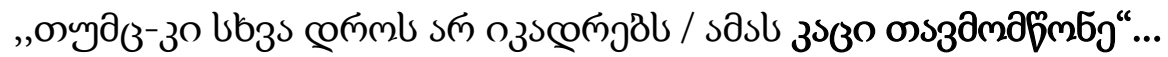

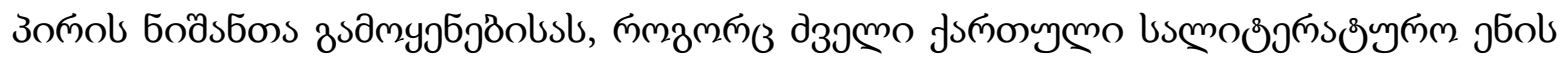

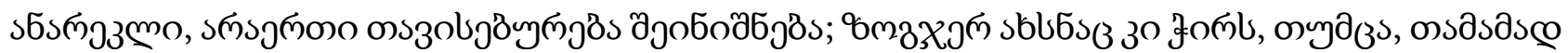

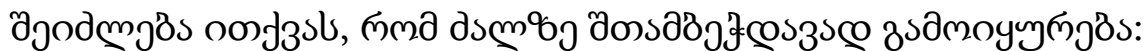

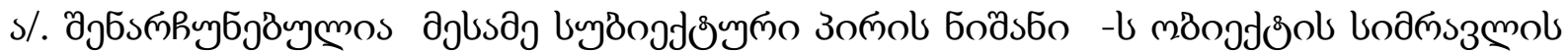

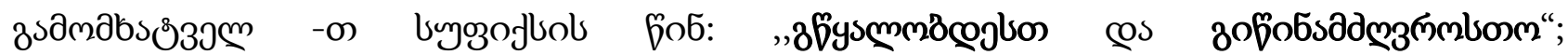

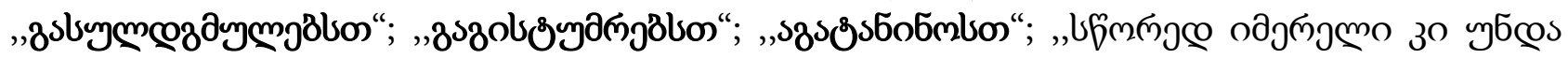

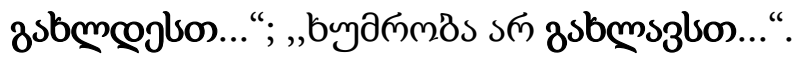

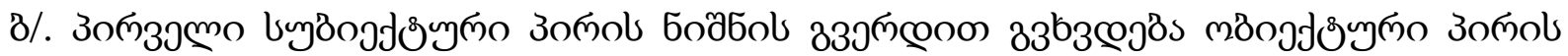

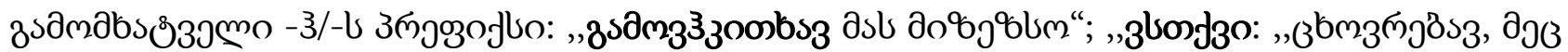

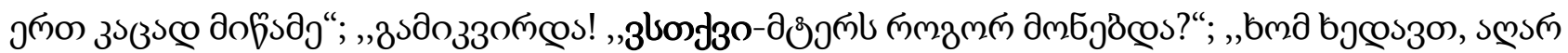

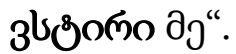

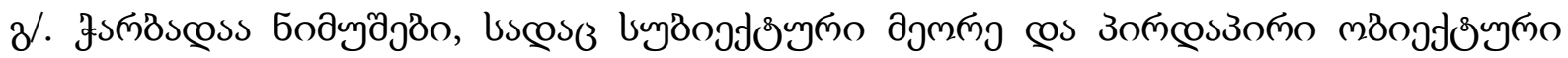

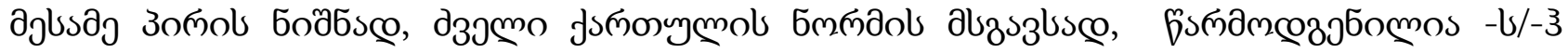

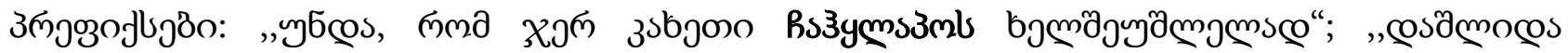

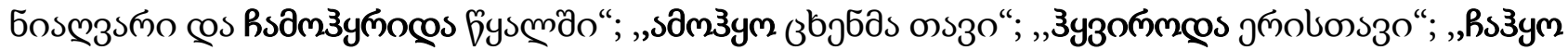

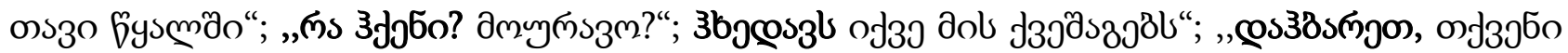

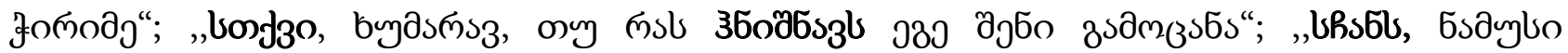

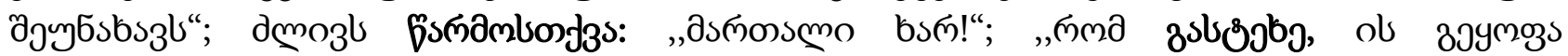

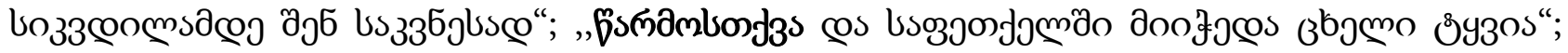

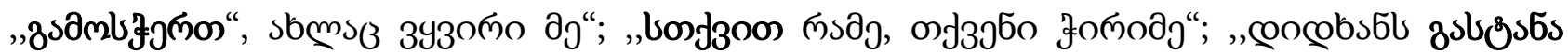




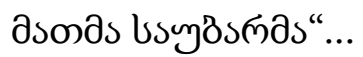

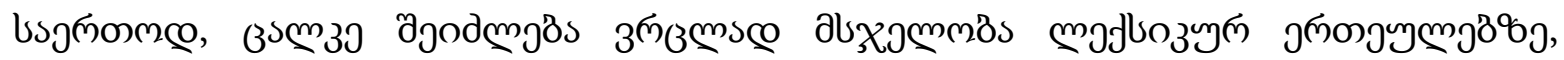

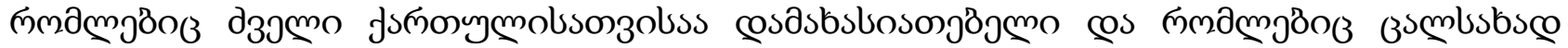
scastsinn

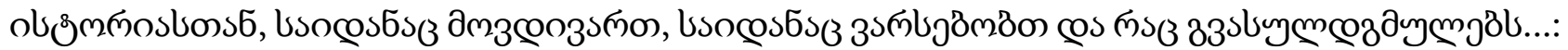

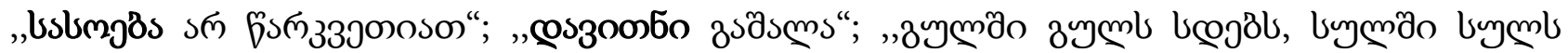

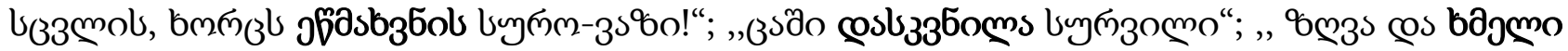

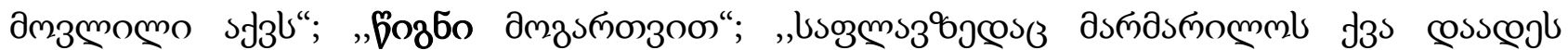

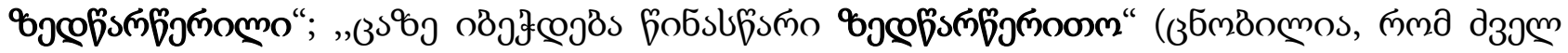

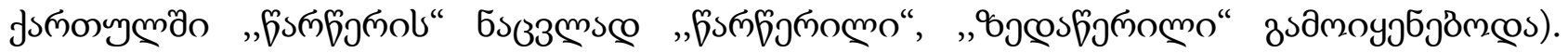

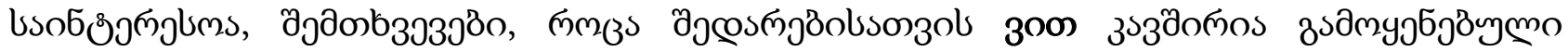

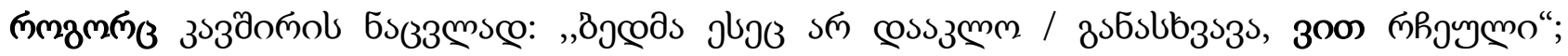

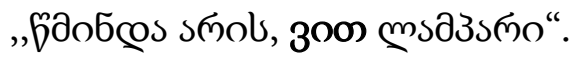

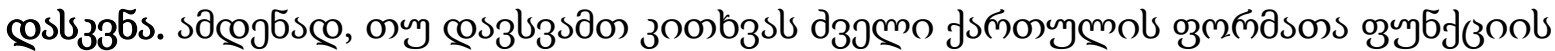

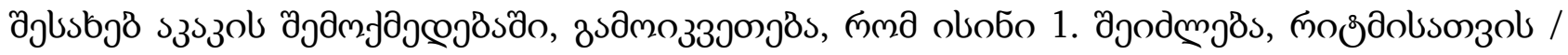

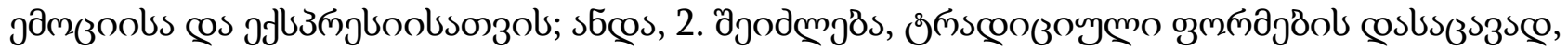

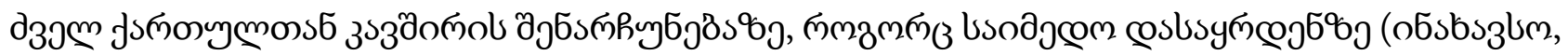

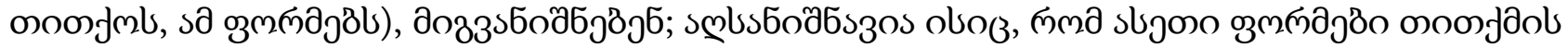

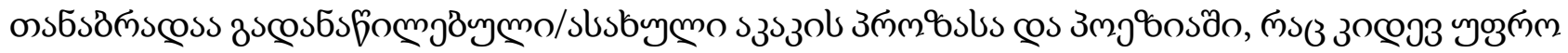

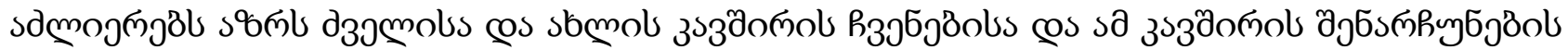

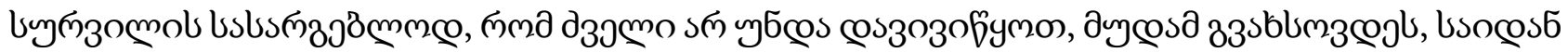

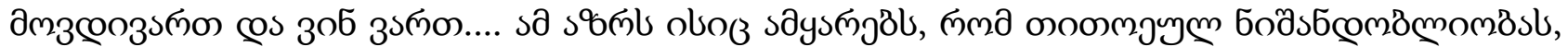

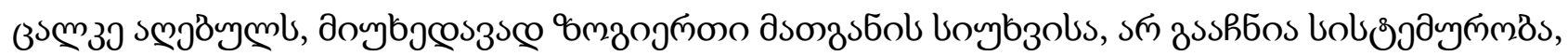

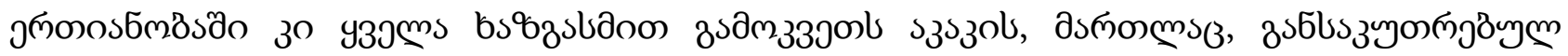

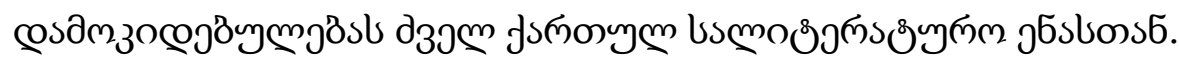

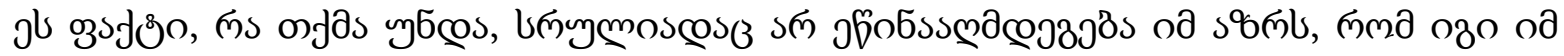

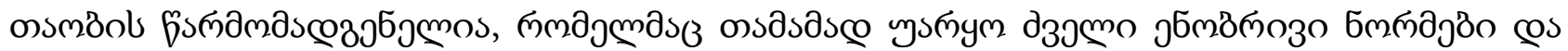

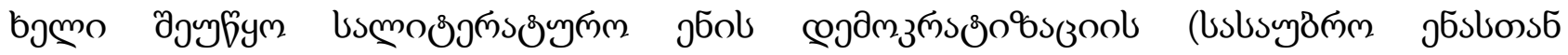

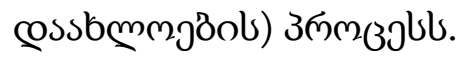

\title{
Characteristics of Old Georgian Literary Language in Akaki Tsereteli's Works
}

Geldiashvili Nunu

Iakob Gogebashvili Telavi State University

\begin{abstract}
Literary Works byAkaki Tsereteli are considered as versatile and diverse. In his works he touches upon almost everything by his poetry, prose, journalism or public work. It is obvious that he established "a type of versatile writer who is equally engaged in prose, poetry, journalism, dramaturgy, translations,
\end{abstract}


children's literature and fables". He was an extremely optimistic person who deeply believed in the future. The following words from one of his works seem amazingly and expressive: "Even if you kill a swallow, Spring will definitely come". Connection between the old and the new forms, that is clearly shown within this emotionally colored expression, has become the goal of the research. We tried to find an answer to the question- what is the role of using old Georgian forms in Akaki's work?!

Given paper analyses the samples such as: 1 . Using proper name by its stem form in nominative case; 2. Ending words by - $\partial s \sigma$ [-man] in the ergative form; 3 . Full stems of demonstrative pronouns - ' $s \partial$ ' [am] , ' $ว$ ' [eg] (=this, that); 4. Using postposition - 'og' [ze] (=on), along with the forms - 'ogœ [-zed] and boœs [-zeda] (=on, over); 5. instrumental case forms formed by a suffix - on [-it] (=with) (without postpositions); 6. Postposition and full agreement of attribute and antecedent 7 . Characteristics of using

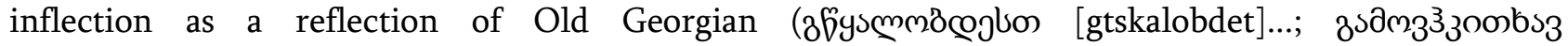

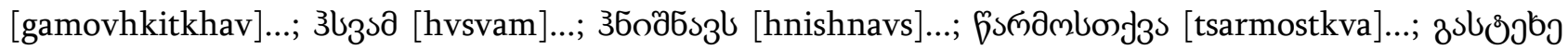
[gastekhe]...); 8. Using conjunction - 3oon [vit] (=as/like) for comparison and so on.

If we ask questions concerning the function of old Georgian forms in Akaki Tsereteli's works, it becomes clear that they can be used for: 1. rhythm, emotiveness and expressiveness; 2. Preserving traditional forms, to maintain the connection between old and new Georgian. It should be mentioned that similar forms are equally reflected in Akaki's prose and poetry which further reinforces the idea in favor of showing the connection between the old and the new and the desire to maintain this connection and always remember where we come from and who we are ... .

This fact does not completely contradict the idea that Akaki is a representative of the generation that courageously rejected the old linguistic norms and contributed to the democratization (rapprochement process with the spoken language) of the literary language.

Key Words: old literary language, characteristics, Akaki Tsereteli. 